\title{
New Resource Allocation Techniques for Base Station Power Reduction in Orthogonal and Non-Orthogonal Multiplexing Systems
}

\author{
Joumana Farah ${ }^{(1)}$, Elie Sfeir ${ }^{(1)}$, Charbel Abdel Nour ${ }^{(2)}$, Catherine Douillard ${ }^{(2)}$ \\ (1) Department of Electricity and Electronics, Faculty of Engineering, \\ Lebanese University, Roumieh, Lebanon \\ (2) Telecom Bretagne, Department of Electronics, Lab-STICC - UMR 6285 \\ Technopôle Brest Iroise, CS 83818 - 29238 Brest Cedex, France
}

\begin{abstract}
This paper provides several new methods for joint subcarrier and power allocation in the contexts of orthogonal and non-orthogonal multiple access. The aim is to minimize the average downlink BS transmit power, under user rate constraints. Particular care is given to the reduction of the complexity of corresponding algorithms. Results show that the proposed methods allow a significant decrease in the necessary total power consumed by NOMA, especially when compared to the case of orthogonal signaling.
\end{abstract}

Index Terms-Energy saving, Orthogonal Frequency Division Multiplexing, Non Orthogonal Multiple Access, Resource allocation, Waterfilling.

\section{INTRODUCTION}

During the last years, the telecommunication sector has witnessed an important proliferation of mobile devices, together with a constantly rising demand for high bandwidthconsuming services. Consequently, the mobile communications community is facing a major problem raised by the increasing amounts of network energy consumption. From the point of view of operators, Base Stations (BS) constitute the main source of power consumption, with more than $70 \%$ of the total amount consumed in mobile networks. It has been estimated that around 3 million of BS, deployed worldwide, consume more than $4.5 \mathrm{GW}$ [1]. From the environmental standpoint, this results in excessive heat dissipation, as well as an important amount of $\mathrm{CO}_{2}$ emissions. From the economic standpoint, this energy consumption yields an important increase in the operational expenditure (OPEX) of mobile operators. All these concerns have been pushing the technical community to find practical solutions that can decrease the energy consumption of mobile networks. Proper energy-efficient algorithms for resource (bandwidth and power) allocation in mobile systems can help to solve these environmental and financial issues. To this end, several strategies have been proposed in the past years, for the reduction of BS power, in the context of downlink Orthogonal Frequency Division Multiplexing (OFDM) systems. One of the first leading solutions was introduced in [2] where a quasi-optimal solution for the power minimization problem was proposed: OFDM subcarriers are first assigned to users based on Lagrange optimization resolved by parameter relaxation. Transmit power and bit loading are then determined by a greedy approach. In [3], a low-complexity method was introduced to reduce the computational load of the solution in [2], by splitting the problem into two phases: resource allocation and subcarrier allocation. Several variants of the suboptimal solution of [3] were proposed in [4-6], which either aim at further reducing its complexity or slightly enhancing its performance. In a previous work [7], we proposed several waterfilling-based greedy solutions for minimizing the spectral occupation of uplink and downlink OFDM systems, while respecting the BS power and data rate constraints of users.

On the other hand, Non-Orthogonal Multiple Access (NOMA) has recently emerged as a potential access scheme for the $5^{\text {th }}$ generation of mobile systems. It consists in exploiting a new domain for user multiplexing, the power domain, by taking advantage of the channel gain difference between paired users on the same subcarrier [8-10]. User separation is done at the receiver side, using Successive Interference Cancellation (SIC). By doing so, NOMA can increase average system throughput by more than $30 \%$ compared to orthogonal signaling, while also improving cell-edge user experience. Also, NOMA implicitly strengthens the fairness between users located in the same cell, and avoids the underutilization of subcarriers experienced when a cell-edge user is scheduled alone using OFDM. However, the design of a scheduler applying nonorthogonal multiplexing needs to be carefully addressed to enable a full exploitation of the NOMA potentials. Indeed, average cell throughput, cell-edge user throughput and user fairness are highly dependent on the way resource allocation (power and bandwidth) is carried out.

In the majority of previous works employing NOMA, the Proportional Fairness (PF) scheduler [11] is adopted, since it provides a good tradeoff between total user throughput and user fairness. Also, fractional transmit power allocation (FTPA) [12] is employed in order to split the power among multiplexed users on the same subcarrier, while equal power allocation is considered between different subcarriers. Despite its multiple advantages, the PF does not allow power minimization, since it aims at striking a balance between total throughput and user fairness, under the constraint of a fixed amount of total BS power. In a former work $[13,14]$, we have proposed several techniques for the minimization of the spectrum occupation in NOMA, under total BS power and data rate constraints. However, because of the difference in problem structure, those techniques cannot be generalized to the case of power minimization. Also, little work has been published that considers the BS power minimization problem with NOMA multiplexing. Two recent letters tackle this problem: In [15], a "relax-then-adjust" procedure is used to provide a suboptimal solution for the NP-hard resource allocation problem. However, the complexity remains relatively high since the algorithm relies on power adjustment and bisection search to resolve the problem of negative powers. In [16], an optimal power allocation technique is first developed for the case of a fixed subcarrier assignment. Then, a deletion-based algorithm is introduced for the joint optimization of subcarrier and power allocation: initially each user is assigned all subcarriers; then, users are iteratively removed from subcarriers until the constraints of the maximum number of multiplexed users are satisfied, thus necessitating a large number of iterations to converge.

In this paper, we aim at introducing novel solutions for joint subcarrier and power allocation that seek the reduction of BS 
power, under user-specific target rate constraints, and with an affordable computational load. In both cases of orthogonal and non-orthogonal signaling, they will incorporate unequal repartition of power between subcarriers. For this purpose, after a brief description of the system model in Section II, we present in Section III a novel solution for power minimization in the context of orthogonal signaling, including a low-complexity recursive waterfilling method. Then, in Section IV, we propose several solutions for extending the study to the case of NOMA signaling. Results and discussions follow in Section $\mathrm{V}$.

\section{SYSTEM DESCRIPTION AND PROBLEM FORMULATION}

Consider a downlink system with $K$ mobile users randomly deployed over a single cell. Each user $k$ requests a transmission rate of $R_{k, \text { req }}$ [bps]. SISO (Single Input Single Output) transmissions are assumed throughout this study. The system bandwidth $B$ is equally divided into $S$ subcarriers. On each subcarrier $n(1 \leq n \leq S)$, a set of $m(n)$ users $\left\{k_{1}, k_{2}, \ldots, k_{m(n)}\right\}$ are chosen from the set of $K$ users, to be scheduled on $n$. Therefore, $k_{i}, 1 \leq i \leq m(n)$, is the $i^{\text {th }}$ user scheduled on $n$ and $m(n)$ the number of users scheduled on $n$. The special case of $m(n)=1$ corresponds to orthogonal signaling.

The signal $x_{n}$ transmitted by the BS on subcarrier $n$ is a superposition of the individual signals of the $m(n)$ paired users:

$$
x_{n}=\sum_{i=1}^{m(n)} x_{k_{i}, n} \text {, with } P_{k_{i}, n}=\mathrm{E}\left[\left|x_{k_{i}, n}\right|^{2}\right] \text { the average transmit }
$$
power of the $i^{\text {th }}$ user on $n$ is.

At the receiver side, the signal of the $i^{\text {th }}$ user detected on subcarrier $n$ can be written as:

$$
y_{k_{i}, n}=h_{k_{i}, n} x_{k_{i}, n}+w_{k_{i}, n},
$$

where $h_{k_{i}, n}$ is the frequency-domain channel coefficient between user $k_{i}$ and the BS over $n$. $w_{k_{i}, n}$ represents the Gaussian noise plus inter-cell interference received by user $k_{i}$ over $n$. In the SIC process, it is assumed that user $k_{i}$ on subcarrier $n$ can remove the inter-user interference from any other user $k_{j}$ whose channel gain verifies $h_{k_{j}, n}<h_{k_{i}, n}$ and treats the received signals from other users as noise.

In order to simplify the study and without loss of generality, in the sequel we will consider the case where the maximum number of paired users per subcarrier is 2 , i.e., $m(n)=1$ or 2 , considering as second (resp. first) user the one having the lower (resp. higher) channel gain between the two users. Indeed, studies such as [17] have shown that the improvement obtained with the scheduling of 3 users per subcarrier, compared to the case with 2 users, is of only $1 \%$ in cell throughput. In addition, ensuring a low number of multiplexed user per subcarrier allows limiting the SIC complexity in the receiver terminals.

Assuming successful decoding and no SIC error propagation in the receiver, and supposing that inter-cell interference is randomized such that it can be considered as white noise [18], the throughput $R_{k_{i}, n}, 1 \leq i \leq 2$, of user $k_{i}$ on $n$ is given by:

$$
\begin{aligned}
& R_{k_{1}, n}=\frac{B}{S} \log _{2}\left(1+\frac{h_{k_{1}, n}^{2} P_{k_{1}, n}}{N_{0} B / S}\right), \\
& R_{k_{2}, n}=\frac{B}{S} \log _{2}\left(1+\frac{h_{k_{2}, n}^{2} P_{k_{2}, n}}{h_{k_{2}, n}^{2} P_{k_{1}, n}+N_{0} B / S}\right),
\end{aligned}
$$

where $N_{0}$ is the power spectral density of the additive white Gaussian noise, including inter-cell interference, assumed to be constant over all subcarriers.

Let $S_{\mathrm{k}}$ be the set of subcarriers allocated to a user $k$ ( $k$ can be either the first or second user on its allocated subcarriers). Our optimization problem can be formulated as follows:

$$
\begin{aligned}
& \left\{P_{k, n}, S_{k}\right\}^{*}=\underset{\left\{P_{k, n}, S_{k}\right\}}{\arg \min } \sum_{k} \sum_{n \in S_{k}} P_{k, n}, \text { subject to: } \\
& \sum_{n \in S_{k}} R_{k, n}=R_{k, r e q}, 1 \leq k \leq K \\
& P_{k, n} \geq 0, \forall n \in S_{k}, 1 \leq k \leq K
\end{aligned}
$$

The problem is combinatorial and non-convex. In the sequel, several allocation techniques will be introduced that seek suboptimal solutions for the assignment problem. The first solutions will be proposed for the orthogonal multiple access (OMA) case, and then for the NOMA scheme.

\section{RESOURCE ALLOCATION SOLUTIONS FOR POWER MINIMIZATION IN ORTHOGONAL MULTIPLEXING}

\section{A. The Rate Craving Greedy (RCG) Solution}

For the OMA case, a well-referenced suboptimal technique was proposed in [3] as an efficient alternative to the highcomplexity Lagrangian Relaxation quasi-optimal solution [2], with results very close to [2]. It consists of two stages: at the resource allocation stage, the average SNR (Signal to Noise Ratio) is computed for each user over all subcarriers. Then, the necessary number of subcarriers is determined for each user based on its requested rate and average SNR. In the subsequent subcarrier allocation stage, the achievable rate is first computed, on each subcarrier, for each user. Rate computation requires the knowledge of the amount of power allocated to each subcarrier. Since users have individual rate constraints, Power Allocation (PA) is carried out, in our implementation of RCG, by applying a waterfilling procedure, separately for each user on all subcarriers, constrained by its requested rate. For this purpose, the gradual dichotomy-based waterfilling approach from [7] is used. Then, each subcarrier is assigned to the user having the highest rate on it. After that, as long as there are users assigned a number of subcarriers different from the one found at the first stage, subcarriers are transferred from users having too many subcarriers to others having less subcarriers than required. These transfers are performed in a way to minimize the rate difference between those subcarriers.

One of the disadvantages of the RCG algorithm is that the number of subcarriers per user is first determined in a separate stage, using the average SNR, which yields a suboptimum solution. In addition, it always uses the whole spectrum, including severely faded subcarriers. For this purpose, we propose several solutions that aim at jointly optimizing the distribution of subcarriers and minimizing the transmit power, so as to reduce the overall BS necessary power.

\section{B. The Maximum user Power - Minimum Decrease of Power solution for Orthogonal Multiple Access (MaxP-MinDP-OMA)}

Minimizing the overall BS power can be achieved in an efficient way by allocating each subcarrier to the user that benefits the most from this allocation, i.e., for whom the power decrease is maximum due to this allocation, under the target per-user rate constraints. However, estimating this power decrease for each candidate user, on the subcarrier $n$ under 
consideration, would necessitate a separate waterfilling procedure for each candidate, including subcarrier $n$ and its formerly attributed subcarriers. This can lead to a prohibitive number of calculations. For this purpose, we start by developing a low-complexity recursive method for estimating the power decrease, in order to incorporate it into our allocation methods.

Let $P_{k, t o t}^{(1)}$ and $P_{k, t o t}^{(2)}$ denote the total amount of necessary power for user $k$, before and after the assignment of a subcarrier to $k$. The amount of power decrease induced by the assignment of subcarrier $n$ to $k$ is $\Delta P_{k, n}=P_{k, t o t}^{(2)}-P_{k, t o t}^{(1)}$. To determine the optimal assignment, PA needs to be applied for each candidate user (for a fixed $n$ ), or for each candidate subcarrier (for a fixed $k$ ), in order to find the corresponding $\Delta P_{k, n}$. In order to limit the computational load of power estimations, we first propose a recursive low-complexity method for calculating the new waterline level after a subcarrier assignment, and the corresponding $\Delta P_{k, n}$.

For a user $k$, the PA over the set $S_{\mathrm{k}}$ of allocated subcarriers can be formulated as the solution of the optimization problem:

$\underset{\left\{P_{k, n}\right\}}{\operatorname{Min}} \sum_{n \in S_{k}} P_{k, n}$, s.t.: $\sum_{n \in S_{k}} \frac{B}{S} \log _{2}\left(1+\frac{P_{k, n} h_{k, n}^{2}}{N_{0} B / S}\right)=R_{k, r e q}$

Using the standard Lagrangian optimization, this leads to the well-known waterfilling solution, where $P_{k, n}$ can be written as:

$P_{k, n}=\max \left\{B \lambda_{k}\left(N_{k}\right) /(S \ln 2)-\left(N_{0} B / S\right) / h_{k, n}^{2}, 0\right\}$,

with $\lambda_{k}\left(N_{k}\right)$ the Lagrange multiplier for user $k$ when the number of its allocated subcarriers is $N_{k}=\operatorname{Card}\left(S_{k}\right)$. The corresponding waterline is: $w_{k}\left(N_{k}\right)=B \lambda_{k}\left(N_{k}\right) / S \ln 2$. By replacing the expression of $P_{k, n}$ into (3), we obtain:

$w_{k}\left(N_{k}\right)=(B / S \ln 2) 2^{\frac{1}{N_{k}}\left[\frac{S}{B} R_{k, \text { req }}-\sum_{n \in S_{k}} \log _{2}\left(\frac{h_{k, n}^{2}}{N_{0} \ln 2}\right)\right] .}$

When a subcarrier $n_{a}$ is added to user $k$, the waterline becomes:

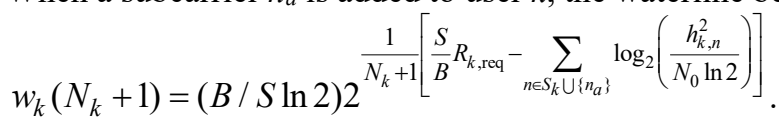

By writing $R_{k, \text { req }}$ in terms of $w_{k}\left(N_{k}\right)$ and replacing it into the expression of $w_{k}\left(N_{k}+1\right)$, we obtain, after some manipulations:

$$
w_{k}\left(N_{k}+1\right)=\left(w_{k}\left(N_{k}\right)\right)^{N_{k} / N_{k}+1} /\left(h_{k, n_{a}}^{2} /\left(N_{0} B / S\right)\right)^{1 / N_{k}+1} .
$$

After the assignment of the first subcarrier $n_{1}$ to $k$, the initial waterline level of user $k$ can be found as the sum of the inverse channel gain and the necessary power to achieve $R_{k, r e q}$ on the subcarrier $n_{l}$ alone. It is therefore:

$w_{k}(1)=\frac{N_{0} B / S}{h_{k, n_{1}}^{2}}+\left(2^{\frac{R_{k, \text { req }}}{B / S}}-1\right) \frac{N_{0} B / S}{h_{k, n_{1}}^{2}}=\frac{N_{0} B / S}{h_{k, n_{1}}^{2}} 2^{\frac{R_{k, \text { req }}}{B / S}}$.

Using (4), it can be verified that adding subcarrier $n_{a}$ decreases the waterline, i.e. $w_{k}\left(N_{k}+1\right)<w_{k}\left(N_{k}\right)$, only if its channel gain verifies the condition: $h_{k, n_{a}}^{2}>\frac{N_{0} B / S}{w_{k}\left(N_{k}\right)}$.

$\Delta P_{k, n}$ can then be obtained by writing $P_{k, \text { tot }}^{(1)}$ and $P_{k, \text { tot }}^{(2)}$ as:

$P_{k, t o t}^{(1)}=N_{k} w_{k}\left(N_{k}\right)-\sum_{n \in S_{k}} \frac{N_{0} B / S}{h_{k, n}^{2}}$,
$P_{k, \text { tot }}^{(2)}=\left(N_{k}+1\right) w_{k}\left(N_{k}+1\right)-\sum_{n \in S_{k} \cup\left\{n_{a}\right\}} \frac{N_{0} B / S}{h_{k, n}^{2}}$, leading to:

$\Delta P_{k, n}=\left(N_{k}+1\right) w_{k}\left(N_{k}+1\right)-N_{k} w_{k}\left(N_{k}\right)-\frac{N_{0} B / S}{h_{k, n_{a}}^{2}}$.

The rest of the section describes our allocation technique for the OMA case. Instead of going over the subcarriers one by one to determine the best user to be assigned on each subcarrier, as was done in most previous works like [2-5, 8, 12, 17, 18], user candidates are first sorted based on a certain priority scheme. Then, at each step of the algorithm, the user with the highest priority is attributed its most favorable subcarrier.

According to Algorithm 1, at each allocation stage, a user $k^{*}$ is first chosen according to a priority assignment procedure. As in $[13,14]$, for the first $K$ assigned subcarriers, priority is set using an initialization phase called "Worst-Best-H" assignment. Its aim is to avoid depriving cell-edge users of their best subcarriers (essential in decreasing their power) in favor of cellinterior users. It proceeds as follows: for each user $k$, the subcarrier with the highest channel gain $h_{k, b e s t}$ is first determined. The user with the lowest $h_{k, \text { best }}$ has the highest priority and is then assigned its best subcarrier. This process is iterated until each user has one allocated subcarrier. At this point, the initialization process is terminated, and priority is now based on the actual necessary transmit power for each user: the user $k^{*}$ with the highest required power is selected to be attributed a new subcarrier from the overall set $S_{p}$ of available subcarriers. $k^{*}$ is assigned the subcarrier $n^{*}$ that leads to the lowest decrease of power. However, only subcarriers verifying (5) are considered as candidates for $k^{*}$. Otherwise, adding this subcarrier to the user would increase its necessary power. Note that, since $k^{*}$ is fixed, minimizing $\Delta P_{k^{*}, n^{*}}$ is equivalent to minimizing the new user's allocated power after subcarrier allocation. On the other hand, it may happen that the allocation of $n^{*}$ to $k^{*}$ decreases its power by a negligible amount. For this purpose, the following condition is tested before subcarrier assignment: $\Delta P_{k^{*}, n^{*}<-} \rho$, where the value of the positive threshold $\rho$ is chosen in such a way to strike a balance between the system power efficiency and spectral efficiency, since unallocated subcarriers may be used by other operators or cognitive systems.

Furthermore, if $\Delta P_{k^{*}, n^{*}>-} \rho, k^{*}$ is removed from the set of users $U_{p}$ whose power level can still be decreased. Before $k^{*}$ is removed from $U_{p}$, we test if $k^{*}$ has a zero amount of power on some of its previously assigned subcarriers (because of the waterline decrease). Such subcarriers are returned to the set of available subcarriers in order to be subsequently allocated. However, such cases rarely occur.

The algorithm stops when no more subcarriers are available or when the set $U_{p}$ is empty. Note that in order to further decrease complexity, and since for all considered subcarriers, $N_{k^{*}} w_{k^{*}}\left(N_{k^{*}}\right)$ is the same when calculating $\Delta P_{k^{*}, n^{*}}$, only the first and third terms in (6) are considered for determining the most favorable subcarrier for user $k^{*}$.

\section{Algorithm 1: MaxP-MinDP-OMA}

$U_{0}$ is the set of users $k$ s.t. $S_{k}=\varnothing$

$H$ is the channel gain matrix with elements $h_{k, n}, 1 \leq k \leq K, 1 \leq n \leq S$

$U_{0}=\{1,2, \ldots, K\} ; U_{p}=\{1,2, \ldots, K\}$ 
$S_{k}=\varnothing, \forall k \in U_{0} ; S_{p}=\{1,2, \ldots, S\}$

// Attribute a subcarrier to each user using the Worst-Best-H priority while $U_{0} \neq \varnothing$ do

$$
\begin{aligned}
& n \_\max _{k}=\underset{n}{\operatorname{argmax}}\left(h_{k, n}\right), k=1, \ldots, K \\
& k^{*}=\underset{k}{\operatorname{argmin}}\left(h_{k, n \_\max _{k}}\right) ; n_{1}=n_{-} \max _{k^{*}} \\
& S_{k^{*}}=S_{k^{*}} \cup\left\{n_{1}\right\} ; S_{p}=S_{p} \cap\left\{n_{l}\right\}^{\mathrm{C}} ; U_{0}=U_{0} \cap\left\{k^{*}\right\}^{\mathrm{C}} \\
& P_{k^{*}, \text { tot }}=\left(2^{R_{k^{*}, \mathrm{req}} /(B / S)}-1\right)\left(N_{0} B / S\right) / h_{k^{*}, n_{1}}^{2} \\
& w_{k^{*}}(1)=\left(N_{0} B / S\right) / h_{k^{*}, n_{1}}^{2}+P_{k^{*}, \text { tot }}
\end{aligned}
$$

\section{end while}

while $S_{p} \neq \varnothing \& U_{p} \neq \varnothing$ do

$$
k^{*}=\underset{k \in U_{p}}{\arg \max } P_{k, \text { tot }} / / \text { identify the user with the highest priority }
$$

for every subcarrier $n \in S_{p}$, s.t. $h_{k^{*}, n}^{2}>N_{0} B /\left(S w_{k^{*}}\left(N_{k^{*}}\right)\right)$ do

Calculate the new waterline $w_{k^{*}}\left(N_{k^{*}}+1\right)$ using (4)

Calculate $\Delta P_{k^{*}, n}$ using (6)

\section{end for}

$$
\begin{aligned}
& n^{*}=\underset{n \in S_{p}, h_{k^{*}, n}^{2}>N_{0} B /\left(S w_{k^{*}}\left(N_{k^{*}}\right)\right)}{\arg \min } \Delta P_{k^{*}, n} \\
& \text { if } \Delta P_{k^{*}, n^{*}}<-\rho \text { do } \\
& S_{k^{*}}=S_{k^{*}} \cup\{n *\} ; S_{p}=S_{p} \cap\left\{n^{*}\right\} \text { C // subcarrier allocation } \\
& P_{k^{*}, \text { tot }}=P_{k^{*}, \text { tot }}+\Delta P_{k^{*}, n^{*}} \quad \text { // power update } \\
& \text { else do } \quad / / \text { removing user and freeing zero-power subcat } \\
& U_{p}=U_{p} \cap\left\{k^{*}\right\} ; \\
& \text { for every subcarrier } n \in S_{k^{*}} \text { s.t. } P_{k^{*}, n}=0 \text { do } \\
& \quad S_{k^{*}}=S_{k^{*}} \cap\{n\} ; S_{p}=S_{p} \cup\{n\} \\
& \text { end for } \\
& \text { end if } \\
& \text { end while }
\end{aligned}
$$

else do // removing user and freeing zero-power subcarriers

\section{RESOURCE ALLOCATION SOLUTIONS FOR POWER MINIMIZATION IN NON-ORTHOGONAL MULTIPLEXING}

The previously proposed OMA resource allocation is next adapted in several ways to the non-orthogonal signaling context. In [13], we have proposed a set of solutions for the spectrum minimization problem in NOMA, under a total power constraint, where PA is performed either by an optimal joint inter and intra-subcarrier PA or by a sub-optimal solution where inter-subcarrier PA is first performed by waterfilling, followed by intra-subcarrier PA using FTPA. The suboptimal PA led to very close results to the optimal one, with a much lower complexity. However, such solutions are not applicable in the power minimization context, since in this case, waterfilling needs to be performed separately for each user such that to meet its rate constraint, with no overall power constraint.

A. The Maximum user Power - Maximum channel gain solution for Non Orthogonal Multiple Access (MaxP-MaxHNOMA)

One of the main difficulties in applying NOMA, while seeking energy reduction, resides in the necessity to meet rate constraints of $K$ independent users, while the achievable rate on each subcarrier depends on the user pairing order (1 or 2) and on the inter-user interference term in the denominator of (2). In other words, each time a new subcarrier is attributed to a user $k$, the amounts of power on its attributed subcarriers need to be jointly estimated with the ones of the users collocated on its subcarriers, so as to respect all rate constraints, as well as the FTPA repartition scheme on each subcarrier [12]:

$P_{k_{2}, n}=P_{k_{1}, n} h_{k_{2}, n}^{-2 \alpha} / h_{k_{1}, n}^{-2 \alpha}$,

with $\alpha$ a decay factor, taken equal to 0.5 in this work.

This would need resolving a non-convex optimization problem at each allocation stage, with a large number of constraints and a prohibitive computational load. Besides, there is no guarantee for converging to a unique optimal solution. A possible iterative approach would be to start by allocating a subcarrier to a user, followed by estimating the minimum power to be consumed on its so far allocated subcarriers so as to meet its rate constraint. This estimate is also dependent on the power attributed in previous steps to its collocating users. Then, the same procedure would be applied to its collocating users, on their corresponding subcarriers, until the power consumption of all users has been updated. The whole process would then be iterated a number of times, until achieving convergence. However, this would induce a large number of chain modifications that need to be performed for each new subcarrier allocation. To counteract this problem, in this first solution, we will operate as follows (Algorithm 2):

First, each user is assigned a subcarrier using the Worst-Best-H criterion. Such subcarriers constitute a subset $S_{W B H}$ of $S_{p}$. Then, at each iteration, the user $k^{*}$ with the highest power is assigned the subcarrier $n^{*}$ with the highest channel amplitude, under the condition: $\Delta P_{k^{*}, n^{*}}<-\rho$.

Let $S_{k}^{\text {sole }}$ be the set of subcarriers where user $k$ is the sole user (i.e. $m(n)=1, \forall n \in S_{k}^{\text {sole }}$ ), $R_{k}^{\text {sole }}$ be the total rate of $k$ on subcarriers in $S_{k}^{\text {sole }}$, and $S_{k}^{\text {first }}$ (resp. $S_{k}^{\text {second }}$ ) be the set of subcarriers where $k$ is first (resp. second) user, collocated with a second (resp. first) one. $R_{k}^{\text {first }}$ and $R_{k}^{\text {second }}$ are the total rates corresponding to $S_{k}^{\text {first }}$ and $S_{k}^{\text {second }}$, found based on (1) and (2). To estimate $\Delta P_{k^{*}, n^{*}}$, the power needed on the subcarriers $S_{k^{*}}^{\text {sole }}$ is first found, so as to reach $R_{k^{*}, \text { req }}$. For this purpose, we take $R_{k^{*}}^{\text {sole }}=R_{k^{*}, \text { req }}-R_{k^{*}}^{\text {first }}-R_{k^{*}}^{\text {second }}$. Then, a gradual waterfilling [7] is performed on the set $S_{k^{*}}^{\text {sole }}$, so as to reach $R_{k^{*}}^{\text {sole }}$, leading to a waterline level $w_{k^{*}}\left(N_{k^{*}}\right)$. Following that, $\Delta P_{k^{*}, n^{*}}$ is found using equations (4) and (6), since $R_{k^{*}}^{\text {sole }}$ is the same before and after adding $n^{*}$ to $S_{k^{*}}^{\text {sole }}$.

If $n^{*}$ is assigned to $k^{*}$ as first user, we search for a potential second user $k_{2}$ to be put on $n^{*}$, unless channel gains of the possible collocating users are close enough to lose the benefits of NOMA. Also, $k_{2}$ must have on $n^{*}$ a channel gain lower than that of $k^{*}$ and is chosen as the one who would benefit the most from this allocation. If $k_{2}$ is assigned to $n^{*}, k^{*}$ and $k_{2}$ will now get fixed rates and powers on $n^{*}$, that will no longer be modified by the algorithm. This fact is essential in avoiding the aforementioned chain of power modifications. At the end of the algorithm, the same steps are applied for the subcarriers in $S_{W B H}$, in order to eventually attribute second users to them. This step was deferred to the end of the algorithm in order to avoid fixing powers earlier on subcarriers in $S_{W B H}$.

\section{Algorithm 2: MaxP-MaxH-NOMA}

Attribute a subcarrier to each user using the Worst-Best-H priority, as in Algorithm 1. 
$S_{p}=S_{p} \cap S_{W B H}{ }^{\mathrm{C}}$

while $S_{p} \neq \varnothing \& U_{p} \neq \varnothing$ do

$k^{*}=\arg \max P_{k, \text { tot }} / /$ identify the user with the highest priority $k \in U_{p}$

$n^{*}=\arg \max h_{k^{*}, n}$ $n \in S_{p}$

Calculate $R_{k^{*}}^{\text {first }}, R_{k^{*}}^{\text {second }}$ and $R_{k^{*}}^{\text {sole }}$

Gradual waterfilling on $S_{k^{*}}^{\text {sole }}$ constrained by $R_{k^{*}}^{\text {sole }} \rightarrow w_{k^{*}}\left(N_{k^{*}}\right)$

Calculate $w_{k^{*}}\left(N_{k^{*}}+1\right)$ using (4) and $\Delta P_{k^{*}, n^{*}}$ using (6)

if $\Delta P_{k^{*}, n^{*}}<-\rho$ do

if $h_{k^{*}, n^{*}}>\left(h_{\max , n^{*}}+h_{\min , n^{*}}\right) / 2$ do $/ /$ search for a second user on $n^{*}$ for every $k_{2}$ in $U_{p}$ s.t. $h_{k_{2}, n^{*}}<h_{k^{*}, n^{*}}$ do

Calculate $R_{k_{2}}^{\text {sole }}$

Gradual waterfilling on $S_{k_{2}}^{\text {sole }}$ constrained by $R_{k_{2}}^{\text {sole }}$ $P_{k_{2}, t o t}^{(2)}=P_{k_{2}, n^{*}}+P_{k_{2}, n\left(n \in S_{k_{2}}^{\text {sole }}\right)}+P_{k_{2}, n\left(n \in S_{k_{2}}^{\text {first }}\right)}+P_{k_{2}, n\left(n \in S_{k_{2}}^{\text {second }}\right)}$ $\Delta P_{k_{2}, n^{*}}=P_{k_{2}, t o t}^{(2)}-P_{k_{2}, t o t}^{(1)} \quad / / P_{k_{2}, t o t}^{(1)}$ is the previous total

\section{end for}

$k_{2}^{*}=\underset{k_{2} \in U_{p}, h_{k_{2}, n^{*}}<h_{k^{*}, n^{*}}}{\arg \min } \Delta P_{k_{2}, n^{*}}$

if $\Delta P_{k_{2}^{*}, n^{*}}<-\rho$ do

$S_{k^{*}}^{\text {first }}=S_{k^{*}}^{\text {first }} \cup\left\{n^{*}\right\} ; S_{p}=S_{p} \cap\left\{n^{*}\right\}^{\mathrm{C}}$

$S_{k_{2}^{*}}^{\text {second }}=S_{k_{2}^{*}}^{\text {second }} \cup\left\{n^{*}\right\}$

Update $P_{k^{*}, \text { tot }}$ and $P_{k_{2}, \text { tot }}$

else do

$S_{k^{*}}^{\text {sole }}=S_{k^{*}}^{\text {sole }} \cup\left\{n^{*}\right\} ; S_{p}=S_{p} \cap\left\{n^{*}\right\}^{\mathrm{C}}$; Update $P_{k^{*}, \text { tot }}$

end if

else do

$S_{k^{*}}^{\text {sole }}=S_{k^{*}}^{\text {sole }} \cup\left\{n^{*}\right\} ; S_{p}=S_{p} \cap\left\{n^{*}\right\}^{\mathrm{C}}$; Update $P_{k^{*}, \text { tot }}$

end if

else do

Free zero-power subcarriers of $k^{*}$ as in Algorithm 1

$U_{p}=U_{p} \cap\left\{k^{*}\right\}^{\mathrm{C}}$; // removing user

end if

\section{end while}

$/ /$ assigning second users to subcarriers in $S_{W B H}$

$U_{p}=\{1,2, \ldots, K\}$

while $S_{W B H} \neq \varnothing \& U_{p} \neq \varnothing$ do

$k_{2}=\arg \max P_{k, t o t}$

for $\forall n \in S_{W B H}$ s.t. $h_{k_{2}, n}<h_{k_{1}, n} \& h_{k_{1}, n}>\left(h_{\max , n}+h_{\min , n}\right) / 2$ do

$/ / k_{l}$ is the first user already assigned to $n$

Calculate $\Delta P_{k_{2}, n}$ //using the previous procedure in Algorithm 2

\section{end for}

$n^{*}=\underset{n \in S_{W B H}, h_{k_{2}, n}<h_{k_{1}, n}, h_{k_{1}, n}>\left(h_{\max , n}+h_{\min , n}\right) / 2}{\arg \min } \Delta P_{k_{2}, n}$

if $\Delta P_{k_{2}, n^{*}}<-\rho$ do

$S_{k_{2}}^{\text {second }}=S_{k_{2}}^{\text {second }} \cup\left\{n^{*}\right\} ; S_{W B H}=S_{W B H} \cap\left\{n^{*}\right\}^{\mathrm{C}}$

Update $P_{k_{2}, t o t}$

else do $U_{p}=U_{p} \cap\left\{k^{*}\right\}^{\mathrm{C}} / /$ removing user

end if

end while
B. The Maximum user Power - Minimum Decrease of Power with Subsequent NOMA solution (MaxP-MinDP-SubNOMA)

In this method, instead of performing the user pairing step directly after the first user assignment, as in Algorithm 2, we first assign first users to all subcarriers (unless this assignment does not yield a significant power decrease). Then, we iteratively identify the user with the highest necessary power and search for the best subcarrier where he could be eventually assigned as second user.

\section{Algorithm 3: MaxP-MinDP-SubNOMA}

$S_{f}$ is the overall set of subcarriers assigned a first user without a second user

Apply MaxP-MinDP-OMA (Algorithm 1)

$U_{p}=\{1,2, \ldots, K\}$

while $S_{f} \neq \varnothing \& U_{p} \neq \varnothing$ do

$k_{2}=\arg \max P_{k, t o t}$

for $\forall n \in S_{f}$ s.t. $h_{k_{2}, n}<h_{k_{1}, n} \& h_{k_{1}, n}>\left(h_{\max , n}+h_{\min , n}\right) / 2$ do

Calculate $\Delta P_{k_{2}, n}$ //using the previous procedure in Algorithm 2

end for

$$
\begin{aligned}
& n^{*}=\underset{n \in S_{f}, h_{k_{2}, n}<h_{k_{1}, n}, h_{k_{1}, n}>\left(h_{\text {max }, n}+h_{\text {min }, n}\right)^{/ 2}}{\arg \min } \Delta P_{k_{2}, n} \\
& \text { if } \Delta P_{k_{2}, n^{*}}<-\rho \text { do } \\
& S_{k_{2}}^{\text {second }}=S_{k_{2}}^{\text {second }} \cup\{n *\} ; S_{f}=S_{f} \cap\left\{n^{*}\right\}^{\mathrm{C}} \\
& \text { Update } P_{k_{2}, t o t} \\
& \text { else do } \\
& \text { Free zero-power subcarriers of } k_{2} \text { as in Algorithm } 1 \\
& U_{p}=U_{p} \cap\left\{k_{2}\right\}^{\mathrm{C}} \\
& \text { end if } \\
& \text { end while }
\end{aligned}
$$

C. The Maximum Power - Minimum Decrease of Power with Subsequent NOMA and Rate Maximization solution (MaxPMinDP-SubNOMA-MaxRate)

In order to reduce the complexity of Algorithm 3, it is modified as follows: in the user pairing step, instead of searching for the subcarrier that leads to the lowest power decrease for user $k_{2}$, we choose the subcarrier $n$ with the maximal achievable rate for $k_{2}$ on $n$. This rate is estimated using (7) and (2) for each candidate subcarrier. Once the subcarrier $n$ has been identified, $R_{k_{2}}^{\text {sole }}=R_{k_{2} \text {,req }}-R_{k_{2}}^{\text {first }}-R_{k_{2}}^{\text {second }}$ is estimated, and a waterfilling is realized on the subcarriers of $k_{2}$ in the set $S_{k_{2}}^{\text {sole }}$ in order to update its total power level $P_{k_{2}, t o t}$.

\section{PRACTICAl Results}

In this section, we assess the performance of the proposed techniques in different practical scenarios. On the one hand, we analyze the impact of using NOMA instead of OMA as a multiplexing scheme. On the other, we show the advantages of our resource allocation techniques towards the RCG method that serves as a benchmark in a plethora of previous works in the field. The following simulation conditions are considered: The cell radius is $500 \mathrm{~m}$ and the system bandwidth $B$ is 10 $\mathrm{MHz}$. The transmission medium is modeled by a frequencyselective Rayleigh fading channel with an rms of $500 \mathrm{~ns}$, and 
distance-dependent path loss with a decay factor of 3.76. Perfect knowledge of the user channel gains by the BS is assumed. The noise power spectral density $N_{0}$ is $4.10^{-18}$ $\mathrm{mW} / \mathrm{Hz}$. Empirical tests (not shown here for the sake of concision) yielded an optimal value for the parameter $\rho$ of 0.01 Watt. At this value of $\rho$, around $90 \%$ of the subcarriers are used by most of our proposed techniques, for 10 users, 128 subcarriers, and with a requested rate of $5 \mathrm{Mbps}$, whereas RCG uses the whole spectrum.

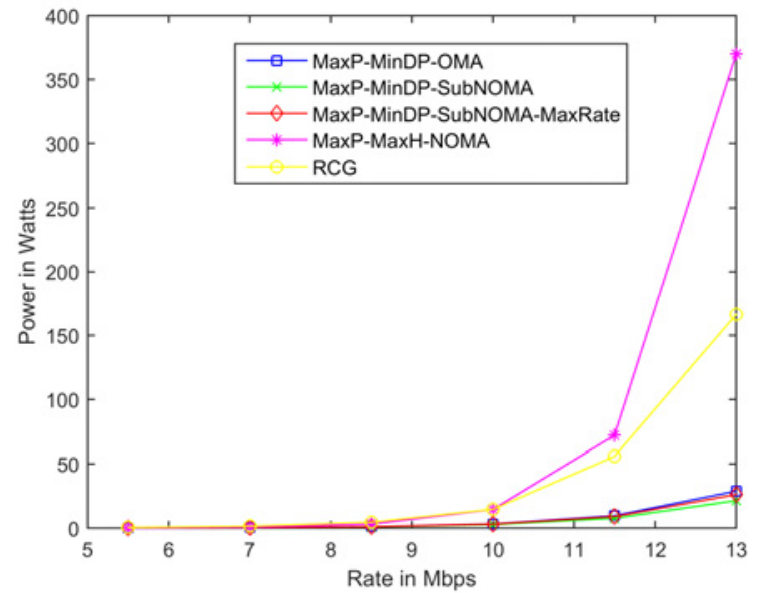

Fig. 1. Total power as a function of $R_{k, \text { req }}$, for $K=10$ and $S=32$.

Fig. 1 shows the total BS transmit power as a function of the user requested rate $R_{k, \text { req }}$, for a number of users $K=10$ and a number of subcarriers $S=32$. The proposed methods, except for MaxP-MaxH-NOMA, clearly outperform RCG, with a gain that increases sharply with $R_{k, \text { req }}$. Starting from a rate of 11.5 Mbps, RCG quickly surpasses the BS power of $40 \mathrm{~W}$ preconized by the LTE (Long Term Evolution) standard [19]. At $R_{k, r e q}=13$ Mbps, MaxP-MinDP-OMA, MaxP-MinDPSubNOMA-MaxRate and MaxP-MinDP-SubNOMA yield respectively $28.80,26.12$, and $21.43 \mathrm{~W}$. This shows the advantage of non-orthogonal multiplexing towards orthogonal multiplexing. This advantage becomes even more important for higher values of $R_{k, \text { req }}$. For instance, at a rate of $14.5 \mathrm{Mbps}$ (not presented on the graph, since the necessary powers of MaxPMaxH-NOMA and RCG become prohibitive), these powers are respectively $86.24,76.05$, and $60.15 \mathrm{~W}$.

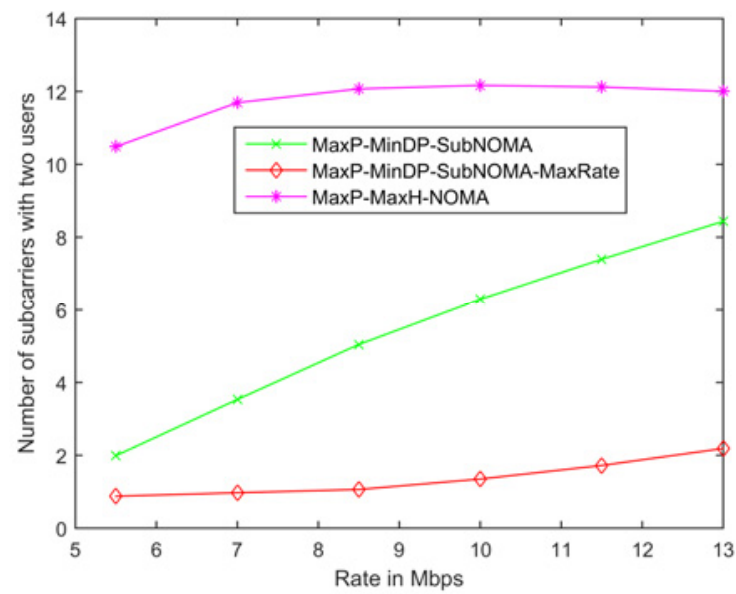

Fig. 2. Number of subcarriers with $m=2$, as a function of $R_{k, \text { req }}$, for $K=10$ and $S=32$

The high power values of MaxP-MaxH-NOMA are due to the fact that at the early iterations of the algorithm, power levels of the first and second NOMA users are high. Besides, powers on subcarriers with two users are fixed in the subsequent steps of the algorithm and can no longer be reduced by waterfilling. On the contrary, in MaxP-MinDP-SubNOMA and MaxP-MinDP-SubNOMA-MaxRate, second users are allocated only after all subcarriers have been assigned first users, i.e., when power levels of first users have been considerably reduced compared to early allocation stages. This leads to lower power levels for second users. Moreover, as shown in Fig. 2, the average number of subcarriers with two users in MaxP-MaxH-NOMA is much higher than that of the two other NOMA methods. It should also be noted that limiting the number of NOMA subcarriers allows reducing the number of SIC operations at the mobile receiver. In the sequel, MaxPMaxH-NOMA results are no longer presented in the graphs.

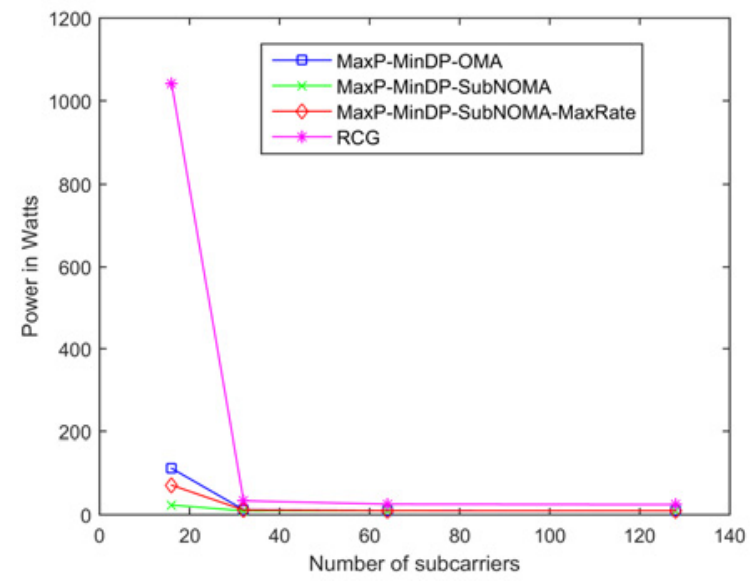

Fig. 3. Total power as a function of $S$, for $K=10$ and $R_{k, r e q}=12 \mathrm{Mbps}$.

In Fig. 3, we show the influence of the number of subcarriers on the necessary amount of BS power. For $K=10$ and $R_{k, \text { req }}=12 \mathrm{Mbps}$, values of $S$ lower than 32 lead to prohibitive amounts of power. Indeed, at $S=16$, the necessary power is 1041.7, 112.2, 71.6, and 23.4 W for RCG, MaxPMinDP-OMA, MaxP-MinDP-SubNOMA-MaxRate and MaxPMinDP-SubNOMA respectively. Beyond $S=64$, the necessary power is almost constant for all methods, around $25.5 \mathrm{~W}$ for RCG and 9.6 $\mathrm{W}$ for the other techniques.

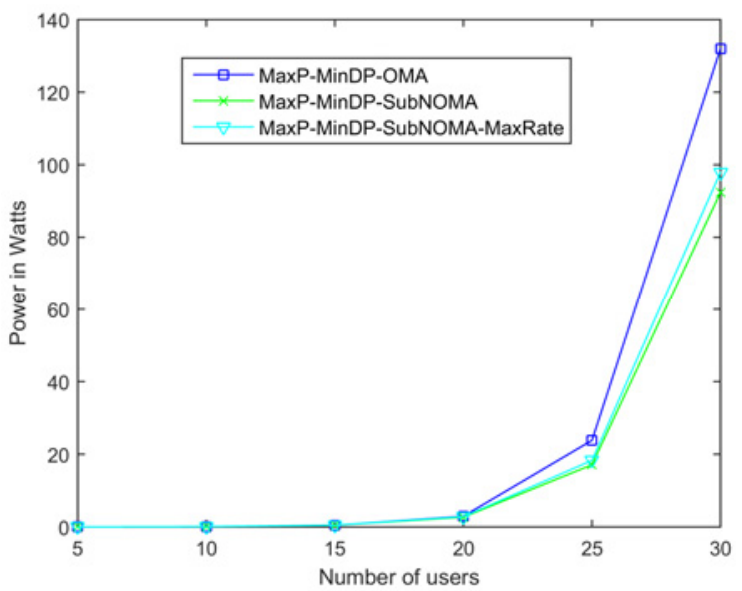

Fig. 4. Total power as a function of $K$, for $S=64$ and $R_{k, \text { req }}=5 \mathrm{Mbps}$.

Fig. 4 presents the total power as a function of $K$, for $S=64$ and $R_{k, \text { req }}=5 \mathrm{Mbps}$. It shows how the gain of MaxP-MinDPSubNOMA towards MaxP-MinDP-OMA and MaxP-MinDPSubNOMA-MaxRate grows quickly as the number of users increases, reaching respectively $40 \mathrm{~W}$ and $6 \mathrm{~W}$ at $K=30$. The measured necessary power with RCG at $K=25$ and 30 is 
respectively 226.8 and $3217.9 \mathrm{~W}$ (not shown in the figure).

As for the difference in performance between MaxPMinDP-SubNOMA-MaxRate and MaxP-MinDP-SubNOMA, it is due to the fact that maximizing the rate of the second user $k_{2}$ on a candidate subcarrier $n$ leads to two contradicting effects: On the one hand, the power of $k_{2}$ tends to be maximized on $n$ (according to (2)), which in turns tends to increase $P_{k_{2}, t o t}$. On the other hand, it tends to minimize the remaining rate $R_{k_{2}}^{\text {sole }}$ to be distributed by waterfilling on the subcarriers in $S_{k_{2}}^{\text {sole }}$, leading to a decrease on the power of the corresponding subcarriers. Therefore, it is generally suboptimum to optimize the user's power by only considering the candidate subcarrier power, without jointly considering the powers of the subcarriers in $S_{k_{2}}^{\text {sole }}$.

TABLE. 1. Average execution time (in $\mathrm{ms}$ ) of one allocation cycle with $R_{k, r e q}=5 \mathrm{Mbps}$.

\begin{tabular}{|l|l|l|l|}
\hline \multirow{2}{*}{ Method } & \multicolumn{3}{|c|}{ Average Execution time [ms] } \\
\cline { 2 - 4 } & $S=32, K=10$ & $S=128, K=10$ & $S=128, K=30$ \\
\hline RCG & 33.80 & 95.22 & 295.44 \\
\hline MaxP-MinDP-OMA & 8.72 & 35.00 & 35.10 \\
\hline MaxP-MaxH-NOMA & 48.58 & 129.61 & 275.97 \\
\hline MaxP-MinDP-SubNOMA & 34.88 & 166.66 & 171.04 \\
\hline MaxP-MinDP-SubNOMA-MaxRate & 14.39 & 48.46 & 67.14 \\
\hline
\end{tabular}

Finally, to get an idea of the computational complexity of the proposed algorithms, Table 1 shows the average execution time of one allocation cycle, measured on a Desktop Intel(R) Core(TM) i5 CPU $2.40 \mathrm{GHz}$. We can clearly see that at a moderate value of $S$, MaxP-MaxH-NOMA has the highest complexity. When $S$ increases, for the same $K$, MaxP-MinDPSubNOMA exceeds the complexity of the other algorithms. In fact, the search for the subcarrier that leads to the lowest power decrease, within the user pairing stage, becomes predominant with respect to the computational load, compared to the other steps of the algorithm. When $K$ increases, for the same $S$, the waterfilling procedures are performed on a smaller number of subcarriers (their approximate average number being $S / K$ ). This explains why the execution time of MaxP-MinDP-SubNOMA becomes less than that of MaxP-MaxH-NOMA and RCG. Regardless of the values of $S$ and $K$, orthogonal signaling remains the least complex, and MaxP-MinDP-SubNOMAMaxRate keeps the lowest complexity among non-orthogonal multiplexing methods. Therefore, in sight of the observed power results in Fig. 1, 3, and 4, this technique presents the best tradeoff between BS power and computational load. However, when allocation techniques are performed at base stations with sufficient computation resources, the utilization of more complex techniques such as MaxP-MaxH-SubNOMA can be justified by the significant gain in transmit power.

\section{CONCLUSION}

In this paper, we propose several resource allocation methods for the minimization of BS transmit power using orthogonal or non-orthogonal multiplexing, under the constraint of user-dependent target rates. Simulation results show substantial gains with NOMA compared to orthogonal multiplexing. An interesting additional result resides in the fact that it seems much more advantageous, from the power perspective, to apply user pairing at a subsequent stage to single-user assignment, i.e., after all subcarriers have been assigned first users, than to jointly assign first and second users. An alternative efficient solution was also proposed to further reduce the complexity of the algorithms, at the expense of a small increase in BS power. We are currently undergoing further research to apply our study in the context of MultipleInput Multiple-Output (MIMO) systems, while taking into consideration the influence of imperfect channel estimation.

\section{ACKNOWLEDGEMENT}

This work has been funded with support from the Lebanese University. Part of this work has been performed in the framework of the Horizon 2020 project FANTASTIC-5G (ICT671660), which is partly funded by the European Union.

\section{REFERENCES}

[1] M. Ismail, W. Zhuang, E. Serpedin, K. Qaraqe, "A Survey on Green Mobile Networking: From the Perspectives of Network Operators and Mobile Users", IEEE Comm. Surveys \& Tutorials, Vol. 17, No. 3, Third Quarter 2015.

[2] C. Y. Wong, R. S. Cheng, K. Ben Letaief, and R. D. Murch, "Multiuser OFDM with Adaptive Subcarrier, Bit, and Power Allocation", IEEE Journal on Sel. Areas in Comm., vol. 17, no. 10, Oct. 1999, p. 1747-58.

[3] D. Kivanc, G. Li, and H. Liu, "Computationally Efficient Bandwidth Allocation and Power Control for OFDMA", IEEE Trans. Wireless Comm., Vol. 2, No. 6, Nov. 2003, p. 1150-58.

[4] Y.-. Lin, T.-H. Chiu, and Y. T. Su, "Optimal and Near-Optimal Resource Allocation Algorithms for OFDMA Networks", IEEE Trans. Wireless Comm., Vol. 8, No. 8, Aug. 2009, pp. 4066-77.

[5] H. Holtkamp, G. Auer, S. Bazzi, and H. Haas, "Minimizing Base Station Power Consumption", IEEE Journal Sel. Areas Comm., Vol. 32, No. 2, February 2014, pp. 297-306.

[6] C. Xiong, G. Y. Li, S. Zhang, Yan C., and S. Xu, "Energy- and SpectralEfficiency Tradeoff in Downlink OFDMA Networks", IEEE Trans. Wir. Comm., Vol. 10, No. 11, 2011, pp. 3874-86.

[7] J. Farah and F. Marx, "Combining strategies for the optimization of resource allocation in a wireless multiuser OFDM system", AEU Int. Jour. of Elect. and Comm., Vol. 61, No. 10, pp. 665-677, 2007.

[8] Y. Saito, Y. Kishiyama, A. Benjebbour, T. Nakamura, A. Li, and K. Higuchi, "Non-Orthogonal Multiple Access for Future Radio Access", Veh. Tech. Conf. (VTC Spring), pp.1-5, 2013.

[9] L. Dai, B. Wang, Y. Yuan, S. Han, C. I, and Z. Wang, "Non-orthogonal multiple access for 5G: Solutions, Challenges, Opportunities, and Future Research Trends", IEEE Comm. Magazine, vol. 53, no. 9, pp. 74-81, 2015.

[10] Z. Ding, P. Fan, and V. Poor, "Impact of user pairing on $5 \mathrm{G}$ nonorthogonal multiple access downlink transmissions", IEEE Trans. on Veh. Tech., vol. 65, no. 8, pp. 6010-6023, Aug. 2016.

[11] M. Kountouris and D. Gesbert, "Memory-based Opportunistic Multi-user Beamforming", Int. Symposium on Information Theory (ISIT), pp. 14261430,2005

[12] A. Benjebbour, A. Li, Y. Saito, Y. Kishiyama, A. Harada, T. Nakamura, "System-Level Performance of Downlink NOMA for Future LTE Enhancements", IEEE Globecom, Dec. 2013.

[13] M.R. Hojeij, J. Farah, C. Abdel Nour, and C. Douillard, "New Optimal and Suboptimal Resource Allocation Techniques for Downlink Nonorthogonal Multiple Access", Wireless Pers. Commun., 2015, pp. 1-31.

[14] M.R. Hojeij, J. Farah, C. Abdel Nour, and C. Douillard, "Resource Allocation in Downlink Non-Orthogonal Multiple Access (NOMA) for Future Radio Access", IEEE Veh. Technol. Conf. (VTC), 2015.

[15] L. Lei, D. Yuan, and P. Varbrand, "On Power Minimization for NonOrthogonal Multiple Access (NOMA)", IEEE Comm. Letters, vol. 20, no. 12 , pp. 2458-2461, 2016.

[16] X. Li, C. Li, and Y. Jin, "Dynamic Resource Allocation for Transmit Power Minimization in OFDM-based NOMA Systems", IEEE Comm. Letters, vol. 20, no. 12, pp. 2558-2561, 2016.

[17] Y. Saito, A. Benjebbour, Y. Kishiyama, and T. Nakamura, "System-level performance evaluation of downlink non-orthogonal multiple access (NOMA)", Int. Symp. on Personal Indoor and Mobile Radio Comm. (PIMRC), 2013, pp. 611-615.

[18] N. Otao, Y. Kishiyama, and K. Higuchi, "Performance of nonorthogonal access with SIC in cellular downlink using proportional fairbased resource allocation", ISWCS 2012, pp. 476-480, 2012.

[19] 3GPP, TR25-814 (V7.1.0), Physical Layer Aspects for Evolved UTRA (2006) 